\title{
Le morse et le phoque dans les mers du Nord au Moyen Âge: chasse, exploitation, commerce. Une approche par les textes
}

\author{
Maxime DELLIAUX \\ Laboratoire H.L.L.I. Histoire, les Langues, les Littératures \& I'Interculturel, \\ Université du Littoral Côte d'Opale, \\ Maison de la Recherche en Sciences humaines, sociales et juridiques, \\ Palais impérial, 17 rue du Puits d'Amour, \\ F-62200 Boulogne-sur-Mer (France) \\ delliaux.maxime@orange.fr
}

Publié le 30 décembre 2016

\section{MOTS CLÉS \\ Morse, \\ phoque \\ techniques de chasse,
commerce, \\ archéozoologie, \\ sagas islandaises, \\ récit Ohthere, \\ Olaus Magnus.}

KEY WORDS

Walrus,
seal,

hunting techniques,

trade,
eology,

zooarchaeology,
Icelandic sagas,

Ohthere's account,

Olaus Magnus.
Delliaux M. 2016. - Le morse et le phoque dans les mers du Nord au Moyen Âge: chasse, exploitation, commerce. Une approche par les textes. Anthropozoologica 51 (2): 85-96. http://dx.doi.org/10.5252/az2016n2a1

\section{RÉSUMÉ}

Les sources dont nous disposons tendent à montrer qu'au Moyen Âge, les hommes craignent la mer et les créatures qui y vivent. Le morse, objet d'une ambiguïté terminologique, n'échappe pas à cette règle. Pourtant, le morse et le phoque sont chassés, les chasseurs ayant une vision plus réaliste de ces animaux. Reposant majoritairement sur des textes nordiques, notamment des sagas islandaises, cet article propose d'identifier les caractères principaux de la chasse dont font l'objet ces deux mammiferes amphibies dans les mers du Nord. Alors qu'elle est vitale dans certaines régions, elle est ailleurs sujette à des règles très contraignantes, qui débouchent parfois sur des conflits. En effet, les mers du Nord sont le théâtre d'un commerce de plus en plus dynamique au cours du Moyen Âge, dans lequel le morse et ses précieuses défenses en ivoire ont leur place.

\section{ABSTRACT}

The walrus and the seal in northern seas during the Middle Ages: hunting, exploitation, trade. A text approach. The sources we have tend to prove that, during the Middle Ages, men fear the sea and the creatures living in it. The walrus, subject of a terminological ambiguity, is no exception to the rule. However, both the walrus and the seal are hunted, for hunters have a more realistic view of these animals. Mainly based on Norse texts, and in particular on Icelandic sagas, this article intends to identify the main features of the hunting of these amphibian mammals in northern seas. While hunting is vital in some areas, it is subject to very restricting rules elsewhere, which can sometimes lead to conflicts. As a matter of fact, northern seas have seen trade become increasingly dynamic during the Middle Ages, in which the walrus and its precious ivory tusks have their place. 


\section{INTRODUCTION}

Nattiqturayuk, «le grand endroit où sont les phoques"; Ugyukhiurvik, «l'endroit où l'on chasse les phoques barbus"; Uqhuquirvik, «l'endroit où l'on transforme la graisse de phoque en huile" (Collignon 1996: 224, 226). Ces trois toponymes inuktitut sont des preuves historiques de la chasse au phoque au Groenland par les Inuit. À travers de tels témoins conservateurs, l'existence de cette pratique ancienne est parvenue jusqu'à nous. Le cas du morse est similaire, comme le relate l'ethnologue Jean Malaurie, spectateur d'une scène de chasse en compagnie d'Inuit (Malaurie 1989: 105-124).

Dans le passé, les hommes du Nord chassaient déjà les mammifères amphibies. Nous le savons notamment par l'archéozoologie (Delort 1984, 1999; Lebecq 2011c). En Scandinavie, durant l'Âge du Bronze (1800-400 av. J.-C.) et l'Âge du Fer (400 av. J.-C. - 800), les populations fabriquaient par exemple de poteries à partir de la graisse de phoque (Siiräinen 2003: 50). De plus, des restes animaliers datant de l'époque médiévale ont été mis au jour en Islande - sites d'Aðalstræti, de Tjarnargata et du Vatnsfjörðr - et au Groenland - sites de Garðar et de Sandnes (Fig. 1; McGovern et al. 1996: 114; Roesdahl 1998a: 19; Perdikaris \& McGovern 2008: 192, $197,198)$. Toutefois, les historiens ne se sont intéressés à la zoohistoire que tardivement (Duchet Suchaux \& Pastoureau 2002:5) et les études sur ce sujet demeurent peu nombreuses. La présente étude vise donc à proposer quelques éléments de réponse, essentiellement à travers l'exploitation de sources textuelles nordiques.

Les morses et les phoques sont des pinnipèdes (Pinnipedia), des mammifères au corps fusiforme et adaptés à la vie marine, leurs pattes s'étant transformées en nageoires. Les morses constituent la famille de Odobenidae et l'espèce des Odobenus rosmarus (Linnaeus, 1758), vivant dans l'espace arctique (voir I.N.P.N.). Ils ont une taille moyenne de 3,5 mètres et un poids moyen de 1,5 tonne. La famille des phoques (Phocidae) qui nous intéresse est celle vivant dans l'hémisphère Nord: les Phocidae phocinae (Linnaeus, 1758). Ce sont des animaux plus petits que les morses, puisqu'ils atteignent en moyenne entre 1,5 et 3 mètres et qu'ils pèsent entre 100 et 400 kilogrammes (Reijnders et al. 1993: 27 ; Roesdahl 1998a: 14; Shirihai \& Jarrett 2011: 307-328, 350).

Ces observations ont été réalisées sur des spécimens contemporains, mais elles sont plus ou moins valables pour le Moyen Âge étant donné que ces animaux n'ont jamais été domestiqués et n'ont pas fait l'objet d'une sélection opérée par l'homme, contrairement au cochon par exemple (Flandrin \& Montanari 1996: 285; Pastoureau 2013: 12-15). En confrontant les données archéologiques relatives aux embarcations utilisées par les Scandinaves installés au Groenland pour aller chasser le morse et les données archéozoologiques concernant l'animal, il semble que, pour un spécimen adulte tué, il reste en moyenne 750 kilogrammes de viande et de graisse et environ 60 kilogrammes de défenses, de mâchoire et de peau (McGovern
1985: 297, 304-305; Charpentier Ljungqvist 2005: 25). En fin de compte, d'après ces calculs, l'animal non décortiqué pouvait bien atteindre environ 1,5 tonne. De manière générale, les chasseurs rapportent dans les Établissements groenlandais (Fig. 1) le crâne de morse avec la mâchoire, la viande et la peau. Compte tenu du temps de navigation nécessaire pour atteindre la zone de chasse comme pour rentrer aux Établissements (en moyenne une quinzaine de jours), on comprend que les chasseurs pratiquent les activités de découpe sur place afin d'optimiser la place disponible dans les embarcations. Cela est d'autant plus important pour eux que la zone de chasse n'est accessible qu'environ sept semaines par an, durant l'été arctique qui s'étend de mi-juin à début septembre (McGovern 1985: 299, 307). In fine, le matériel archéozoologique disponible est très mince, ce qui rend difficile toute approche biologique des animaux de l'époque médiévale.

Le morse et le phoque ont été étudiés en premier lieu à travers le prisme de l'activité cynégétique. Il convient également d'analyser les utilisations matérielles qu'ont pu en faire les hommes. La dimension économique occupe en effet une place importante dans notre réflexion. Durant les premiers siècles du Moyen Âge, le centre de gravité du commerce se déplace de l'espace méditerranéen vers l'espace nordique. Dès le IXe siècle, les Frisons sont présents dans la mer Baltique (Lebecq 1983: 30, 31). La pénétration frisonne en Europe du Nord est capitale afin de comprendre les origines du phénomène viking, borne chronologique haute de cette étude. L'appétit scandinave a été attisé par la vue de richesses variées apportées par les Frisons et les Anglo-Saxons, qui ont fait connaître aux Scandinaves les grands axes marchands et les ont initiés à la navigation à voile (Lebecq 2011a: 138), l'ivoire de morse trouvant sans difficulté sa place dans ce nouveau système économique.

Ce travail a pour terminus ante quem les œuvres de l'archevêque suédois Olaus Magnus (XVIe siècle). Diplômé de l'université de Rostock où il a développé son goût pour les sciences naturelles, il entre en Suède dans les années 1510 et voyage dans le nord de la Scandinavie. Son attention portée aux détails pittoresques rend ses ouvres singulièrement riches, à commencer par la Carta marina (1539) et l' Historia de gentibus Septentrionalibus (1555), immense commentaire de ladite carte dans lequel l'auteur mêle compilation des auteurs classiques et médiévaux ainsi que connaissances personnelles issues de son expérience de Scandinave (Maillefer 2004: 9-15; Delliaux 2015). Par exemple, Olaus Magnus est l'un des premiers auteurs à avoir proposé une description du morse.

Notre propos s'étend donc sur plus de sept siècles et s'appuie avant tout sur des sources textuelles, auxquelles viennent répondre des éléments iconographiques et archéozoologiques. Nous présenterons brièvement ces sources et leur intérêt dans une première partie. Létude de la chasse, des acteurs qu'elle implique, des lieux où elle se déroule ainsi que ses techniques et difficultés constituera la deuxième approche de notre réflexion. Dans un dernier temps, il s'agira pour nous d'étudier l'exploitation et le commerce qui sont faits du morse et du phoque. 


\section{PRÉCISIONS SUR LES SOURCES}

Les sources textuelles qui ont été dépouillées sont des sources nordiques, en particulier des sögur [sagas] du XIIIe siècle. Il s'agit de récits en prose avec un fond plus ou moins historique et rapportant la vie et les faits et gestes d'un personnage digne de mémoire (Boyer 2001b: 189). Snorri Sturluson figure parmi les sagnamenn [auteurs de sagas] les plus connus; il a étudié à l'école d'Oddi, l'un des centres culturels les plus prestigieux de l'Islande (Boyer 2001b: 61-64). Il sera fait référence à cinq sagas ainsi qu'à un dit, c'est-à-dire un texte bref ayant toutes les caractéristiques de la saga. Parmi ces sources nordiques, n'oublions pas le Landnámabók [Livre de la colonisation de l'Islande], qui fait le récit de l'installation des premières familles scandinaves en Islande (Cattanéo 2013b), ainsi que la Konungs skuggsjá [Miroir royal]. Cette source est un texte détaillé rédigé par un Norvégien anonyme dans la seconde moitié du XIIIe siècle. L'espace septentrional y est décrit avec un objectif politique: le roi de Norvège Hákon IV (12171263) souhaitait en effet établir son autorité sur l'Islande et le Groenland (Jónsson 1997: 13-16). La Konungs skuggsjá est donc une manière pour lui de légitimer par la connaissance géographique son pouvoir sur ces territoires septentrionaux. Si ces documents présentent l'avantage de nous offrir un point de vue nordique sur les animaux, ils ne sont toutefois que d'une utilité limitée, car leur intérêt premier n'est pas le monde animal. En effet, à l'exception de la Konungs skuggsjá, il s'agit plutôt de textes épiques, où l'aventure constitue le cœur du récit.

Afin de pallier cette lacune, un ouvrage encyclopédique sera sollicité de manière épisodique: l'Historia de gentibus Septentrionalibus d'Olaus Magnus. Les connaissances universelles y sont répertoriées, ce qui permet de dresser le panorama du savoir à un moment donné. Les travaux d'Olaus Magnus - sa Carta marina et son Historia - ont indéniablement permis de sortir le Nord de certains des stéréotypes qui l'emprisonnaient, mais les monstres marins demeurent des objets stimulant l'imagination. À la lecture de l'Historia, le lecteur rencontre dans les eaux islandaises des créatures à la cruauté indomptable ("indomabilis immanitas») ou d'effroyables monstres ("horribilia monstra») le long des côtes norvégiennes, dont la pupille rouge, telles des flammes, est perceptible par les pêcheurs lorsque le ciel est obscur (Olaus Magnus 1998: xxi 3 ; xxi, 5). De même, les marins des mers septentrionales redoutent la baleine naufrageuse car, d'après la description qu'Olaus Magnus en donne, elle s'écrase de tout son poids sur les navires et les submerge (Olaus Magnus 1998: xxi, 7). Des vignettes de l'Historia de gentibus Septentrionalibus proposent d'ailleurs des représentations de ces monstres, ce qui participe du succès de l'œuvre (Ribémont 1997: 102-103; Balzamo 2009: 122). En ce qui concerne les monstres, Olaus Magnus s'intègre pleinement dans le long Moyen Âge de Jacques Le Goff. Les peurs et superstitions médiévales perdurent au XVIe siècle. Ce n'est que dans le second XIXe siècle et plus encore au XXe siècle que les découvertes scientifiques tendent à rationnaliser l'univers maritime et à en marginaliser le pan merveilleux et/ou monstrueux (Corbin 2004: 13).

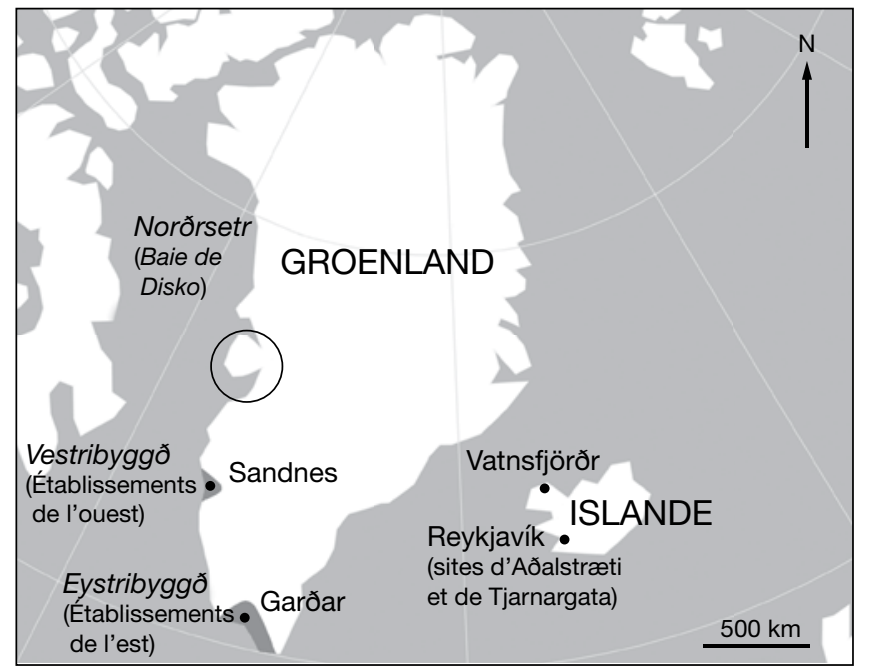

FIG. 1. - Localisation des Norðrsetr et des sites archéologiques en rapport avec le morse et/ou le phoque.

Quant aux textes juridiques, ils nous renseignent sur les normes en vigueur à l'époque où ils ont été consignés. Pour l'Islande, il existe la Grágás, un code juridique constituant une collection d'anciennes et de nouvelles lois. Elles ont été compilées dans les années 1140 et probablement utilisées jusqu'en 1262-1264 (Holman 2003: 171; Cattanéo 2013a). C'est à cette date que l'île passe sous l'autorité de la Couronne norvégienne. Concernant la Norvège, nous disposons de la loi du Gulaping, promulguée dans les années 1270 , mais qui contient des dispositions remontant parfois au XIIe siècle (Holman 2003: 172). La loi de la Jónsbók concerne également la Norvège. Compilée au XIIIe siècle, elle a été acceptée par les Islandais en 1281 (Byock 2007: 378). En dépit de leur organisation très formelle, ces textes sont extrêmement instructifs, en particulier sur les questions cynégétiques et alimentaires.

Enfin, il est nécessaire d'accorder au récit d'Ohthere une place particulière. Originaire du Hålogaland, la province la plus septentrionale habitée par les Norvégiens, Ohthere a entrepris dans les années 870-890 un voyage jusqu'en mer Blanche. Il a ensuite été reçu à la cour du roi Alfred de Wessex (871-899), où il a raconté son voyage, qui a alors été mis par écrit (Lebecq 2011b: 225-227). Ce récit est précieux pour nous, car Ohthere explique avoir rencontré des horshwal. Ce zoonyme vieil-anglais composé des racines hors [cheval] et hwal [baleine] correspond au morse, la baleine-cheval.

L'exposé qui suit repose prioritairement sur des sources écrites. Les données archéologiques n’ont pas été exclues pour autant: elles ont parfois été mises en valeur, essentiellement pour confirmer les tendances qui se dégagent à la lecture des textes; quelques sites archéologiques ont par exemple été mentionnés dans les pages précédentes. Nous ferons de temps en temps appel à d'autres témoins archéologiques, venant cette fois combler les lacunes des sources écrites. Une exploitation plus approfondie de ces données reste néanmoins souhaitable et nécessaire afin d'approfondir la réflexion. 


\section{LA CHASSE AU MORSE ET AU PHOQUE}

Les anthroponymes zoophores présents dans les sagas islandaises interrogent sur la chasse. Au fil de la lecture de ces sources, on rencontre des personnages comme Helgi selseista, c'est-à-dire «Helgi Testicule-de-Phoque» (Boyer 1987e: 678) ou Sela-Kálfr, «Kálfr-aux-Phoques» (Boyer 2000: 158). Les exemples semblables ne manquent pas, chaque saga apportant son lot de sobriquets. Que traduisent ces spécificités anthroponymiques? S'agit-il de noms ironiques? Ces hommes étaient-ils des chasseurs de phoque ou des commerçants? Nous ne pouvons pas répondre avec une certitude absolue à ces interrogations, mais il apparaît clairement que l'animal occupe une place importante dans les pratiques sociales, que les sagnamenn relatent d'ailleurs sans hésitation, dans un souci d'authenticité.

\section{Prélude TERMINOLOGIQUe:}

LE MORSE EST-IL UN MONSTRE?

Le morse tel qu'il est présenté dans les sources écrites est un cas particulier. François-Xavier Dillmann a montré que plusieurs zoonymes existent dans les textes: hrosshvalr, rosmal et rostungr. Cependant, ils ne font pas tous référence au pinnipède. En effet, le hrosshvalr ne semble pas être un morse (Dillmann 2006: 248-255).

Le zoonyme le plus ancien concernant le morse semble être le horshwal d'Ohthere. Les deux racines du terme vieilanglais - hors et hwal-se retrouvent dans l'appellation norroise hrosshvalr avec hross [cheval] et hvalr [baleine]. Littéralement, ces animaux sont des baleines-cheval. Dans l'Historia Norwegia, brève histoire de la Norvège rédigée au plus tard dans les années 1220 par un moine anonyme (Faulkes \& Perkins 2001: xi), la baleine-cheval apparait également, sous la forme latine equinus cetus (Dillmann 2006: 255).

La baleine-cheval est donc présente dans les sources, et cela du vieil-anglais au latin en passant par le vieux-norrois. Le hrosshvalr serait l'équivalent du horshwal. Ohthere dit qu'il s'est rendu chez les Finnas "pour les "baleines-cheval" (horshwal), parce qu'elles [ont] dans leurs défenses un excellent ivoire" (Lebecq 2011b: 234). Au vu de cette description, le horshwal est bien un morse. Le terme vieil-anglais est peut-être issu du vieux-norrois hrosshvalr (Horrent 1946: 69). Le navigateur norvégien aurait rapporté avoir vu des hrosshvalr en mer Blanche et ses auditeurs du Wessex, ignorant l'existence d'un tel animal, auraient transcrit le mot scandinave dans leur langue, donnant horshwal. L'hypothèse contraire est peu probable car, étant donné le contexte géographique des îles Britanniques, les Anglais ne connaissaient vraisemblablement pas la baleine-cheval avant qu'Ohthere ne vienne à leur rencontre.

Pourquoi le morse est-il appelé baleine-cheval? Une telle dénomination n'est pas due au hasard. Au vu des éléments dont nous disposons, nous pouvons dire qu'à une époque antérieure aux années 870-890, des Scandinaves ont attribué à l'animal le nom hrosshvalr, sans doute parce qu'ils ont remarqué des similitudes entre ces animaux, dans une logique comparable à celle de Pline l'Ancien, pour qui chaque animal marin a son équivalent terrestre (Thomasset 2002: 61). Comparer le morse au cheval tient peut-être à l'utilisation qui peut être faite de son cuir pour fabriquer des cordes, comme le signale la Konungs skuggsjá (Jónsson 1997: 69). Par ailleurs, son allure massive et son besoin d'eau pour survivre peuvent expliquer la comparaison avec la baleine.

Le zoonyme hrosshvalr serait donc l'un des plus anciens termes désignant le morse. Toutefois, dans les lois de la Grágás, le morse (rosmal) et la baleine-cheval (roshval) sont clairement différenciés (Dennis et al. 1980: 50), ce que confirme d'ailleurs la Konungs skuggsjá, où le morse est nommé rostungr, tandis que le hrosshvalr est un adversaire de l'homme:

"Puis il y a les espèces de baleines qui sont féroces et cruelles envers les hommes et cherchent à perdre les hommes partout où elles le peuvent: l'une s'appelle hrosshvalr (baleine-cheval) et l'autre raudkembingr (peigne-rouge). Celles-ci sont pleines de voracité et de méchanceté; jamais elles ne sont rassasiées de tueries, car elles parcourent toutes les mers pour chercher si on trouve des bateaux. Puis elles sautent pour pouvoir les couler plus vite et les perdre de cette façon. Les hommes ne mangent pas ces baleines, car elles sont leurs adversaires, comme si elles étaient déterminées [à] être des ennemis du genre humain" (Jónsson 1997: 50).

Avec le temps, du moins entre l'époque d'Ohthere (deuxième moitié du IXe siècle) et celle de la mise par écrit de la Grágás (XIIe siècle), la confusion s'est développée, rendant opaque la distinction entre les étymons norrois. Les sources s'accordent en tout cas sur un point: le hrosshvalr n'est pas un morse.

Au XVIe siècle, Olaus Magnus ajoute de l'ambiguïté. Dans son Historia de gentibus Septentrionalibus, il décrit le morse en employant le terme latin rosmarus, que l'on peut rapprocher du vieux-norrois rosmal désignant bien le pinnipède. L'animal est aussi nommé morsus par l'humaniste suédois. L'utilisation de la conjonction de coordination sive (ou inclusif latin) dans le titre "De rosmaro sive morso Norvagico" (Olaus Magnus 1998: xxi, 28) montre que le rosmarus et le morsus sont un seul et même animal dans l'œuvre d'Olaus Magnus. Néanmoins, l'auteur justifie le zoonyme morsus par le verbe mordere [mordre]. D'après la description qu'il en donne, le morse a des dents tranchantes (en fait, ses défenses) avec lesquelles il attaque l'homme:

«Norvagicum littus versus loca ad Septentrionem magis vergentia, maximos ac grandes pisces elephantis magnitudine habet, qui morsi seu rosmari vocantur, forsitan ab asperitate mordendi sic appellati" (Olaus Magnus 1998: xxi, 28).

En proposant cette explication étymologique, Olaus Magnus fait du morse un animal dangereux. Le spectre du redoutable hrosshvalr réapparaît donc. Le morsus olavien est en effet capable d'escalader les falaises et il attaque l'homme (Olaus Magnus 1998: xxi, 28). Ses canines orientées vers le haut sont une arme redoutable (Fig. 2). Mais dans le même temps, en décrivant 
un tel monstre, l'auteur insiste sur les mirabilia nordiques et fait du Septentrion un univers onirique, à la frontière entre réalité et fiction (Johannesson 1991: 177). Le discours d'Olaus Magnus prend davantage en compte le morse pour l'image et le message qu'il véhicule que pour l'animal en soi. Ce procédé n'est pas nouveau, puisque les bestiaires médiévaux sont déjà bâtis sur cette conception de la faune (Voisenet 2000: 324, 325; Duchet Suchaux \& Pastoureau 2002: 7). En revanche, la vision que les chasseurs ont des animaux est plus pragmatique et démystifiée, car ils sont en contact direct avec leur proie, en particulier lors de la chasse au harpon.

\section{LES ACTEURS ET LES ZONES DE CHASSE}

Les sagas islandaises sont des textes qui nous intéressent pour cette question. La chasse n'est pas au cœur des préoccupations des sagnamenn, mais de maigres détails sont souvent distillés dans ces textes afin d'introduire de la couleur locale et de recréer l'univers mental passé tel qu'ils l'imaginent. Les sagnamenn écrivent en moyenne deux siècles après les événements qu'ils relatent et font de facto de la reconstitution historique (Boyer 2012: 110). Partant de faits, ils reconstruisent le passé, injectant au passage des souvenirs, traditions et légendes (Boyer 2012: 111).

Malgré leur silence relatif (Barbarini 1990: 78), les sagas livrent donc quelques éléments. La chasse ne semble pas nécessiter une logistique et un équipement conséquents; certaines techniques présupposent néanmoins la détention d'un bateau. Par exemple, dans les Annales du Groenland, sont décrits des bateaux à six rames faisant au printemps le voyage des Établissements, au sud de l'île, vers les zones de chasse, plus au nord (Perdikaris \& McGovern 2008: 192). En revanche, d'autres techniques cynégétiques sont moins contraignantes et ne nécessitent pas l'emploi d'une embarcation. Citons par exemple la technique consistant à frapper les phoques avec un gourdin lorsqu'ils sont sur la terre ferme (Ogilvie et al. 2009: 12).

De plus, la chasse n'est pas l'apanage des élites sociales, tout individu libre pouvant chasser. Sur le plan socio-juridique, le propriétaire de la zone de chasse a de nombreux privilèges par rapport aux chasseurs. Par exemple, la loi du Gulaping stipule que si un animal est repéré dans un piège ou dans la mer à proximité du rivage, il revient de droit au propriétaire de la zone de chasse en question (Larson 2008: 102). Théoriquement bien public, le rivage appartient en réalité au propriétaire du terrain contigu (Boyer 1987b: 54). Dans ce contexte, si un individu découvre un phoque piégé dans la propriété d'un autre individu, il doit le tuer et l'amener au propriétaire (Larson 2008: 102). Des conflits émergent parfois de cette législation au nom du rekatháttr, le droit des épaves (Boyer 1987d: 311). Cependant dans les almenningar, les portions de littoral collectives, la législation ne prévoit rien et la récupération d'une reki [épave] tourne parfois à la tragédie (Boyer 1987e: 654-655).

Malgré quelques contraintes juridiques, l'essentiel de la population - les hommes libres - est autorisé à chasser. Reste néanmoins à savoir où la chasse était pratiquée. Au IXe siècle, le Norvégien Ohthere confie à ses interlocuteurs anglais

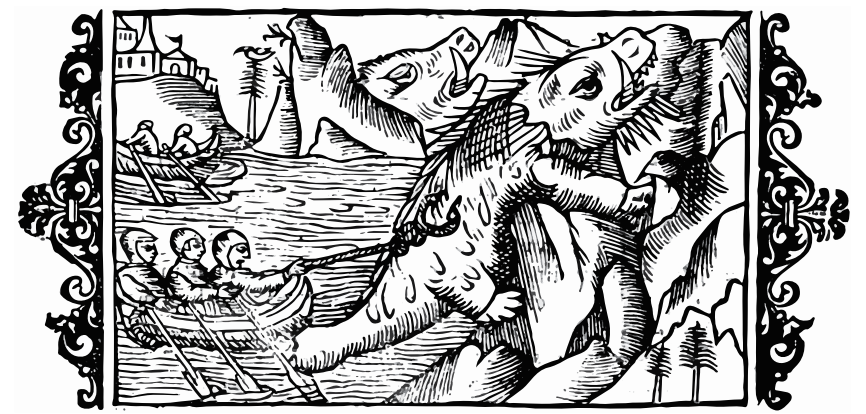

FIG. 2. - Olaus Magnus, Historia de gentibus Septentrionalibus, xxi, 28. Vignette extraite d'un exemplaire de l'editio princeps (1555), numérisé en 2010. Bibliothèque de l'université de Gand, Belgique.

que les baleines-cheval ont dans leurs défenses un ivoire de très bonne qualité et que leur peau est bonne pour faire des cordages de navires: "hiora hid bið swiðe god to sciprapum" (Lebecq 2011b: 234; Lebecq 2011c: 242). D'après ce récit, le morse est chassé en mer Blanche. En effet, durant son voyage, Ohthere franchit le cap Nord et arrive chez les Finnas, c'està-dire les Lapons (Valtonen 2007: 106; Lebecq 2011b: 234), qui d'après lui chassent le morse. Ohthere ramène d'ailleurs des canines au roi Alfred de Wessex (Lebecq 2011b: 225). Cette géographie est corroborée par l'archéologie, puisque des installations, dont la date fluctue entre le VII e et le Xe siècle et servant pour l'extraction de l'huile des mammifères marins ou amphibies, ont été découvertes sur le littoral arctique. Elles témoignent par conséquent de l'activité cynégétique dans la région (Storli 2007: 91).

Le morse intéresse à un tel point les Norvégiens que les jarls (grands) se querellent, chacun voulant prélever le tribut sur les Finnas (Olsen 2003: 16). Ainsi, dans la Saga d'Egill, fils de Grimr le Chauve, il est fait mention à plusieurs reprises du monopole du commerce et de l'impôt des Norvégiens sur les Lapons (Boyer 1987b), peut-être les populations de la péninsule de Kola (Lebecq 2011b: 227, 229, 230). Il s'agit d'un objet de différends, ce qui révèle peut-être l'importance des produits de luxe issus de l'animal et donc, en amont, de sa chasse. Les sagas, dont l'historicité peut être questionnée, sont néanmoins validées par le récit d'Ohthere, qui a exploré le nord de la Scandinavie à la recherche des horshwal.

Pour ce qui est de l'Islande, des données archéozoologiques témoignent d'une chasse au morse qui s'inscrit dans la durée. À Aðalstræti, des côtes et défenses de morse datant de la fin $\mathrm{du} \mathrm{IX}^{\mathrm{e}}$ siècle et du début du Xe siècle ont été découvertes (Frei et al. 2015: 442); sur le site de Tjarnargata, ce sont un fragment de crâne du IXe-XIIIe siècle et une vertèbre du XIIIe-XVe siècle qui ont été mis au jour (Frei et al. 2015: 443). Ce matériel permet de valider le sens du toponyme Rosmhvalanes ("Cap-des-morses») apparaissant à quatre reprises dans le Landnámabók (Boyer 2000: 52, 112, 258, 260). Ce cap (nes) se situe à l'extrémité de la péninsule de Reykjanes, au sud-ouest de l'Islande. Dès la fin du XIXe siècle, des crânes de morses y avaient été découverts, ce qui concordait déjà avec le toponyme (Pierce 2009: 57). Le morse a bien été présent en Islande, mais cela ne nous permet pas d'en déduire 
une chasse d'ampleur, tout au plus un abattage occasionnel (Pierce 2009: 55).

Le pinnipède est également chassé au Groenland: la petite pierre runique de Kingigtorssuaq $(10 \times 3 \mathrm{~cm})$, à l'ouest de l'île, en témoigne particulièrement. Découverte en 1824 mais perdue à ce jour, elle est une preuve de la présence scandinave à Kingigtorssuaq (sur l'île de Kingigtortagdlit), les runes étant l' «unique outil d'expression graphique des Vikings» à partir du IXe siècle (Marez 2007). Plus largement, cette pierre atteste leur présence dans les Norðrsetr, littéralement les lieux (setr) au Nord (Norðr). Cette zone de chasse au nord des Établissements groenlandais correspond à l'actuelle baie de Disko (Fig 1) et fut une destination privilégiée des Scandinaves (Charpentier Ljungqvist 2005; Frei et al. 2015: 445). Datant probablement du XIVe siècle, la pierre prouve que les Scandinaves chassaient au nord des Établissements à cette époque; il est probable qu'ils pratiquaient déjà cette activité avant le XIVe siècle (McGovern 1985: 284). Les sources textuelles disponibles nous permettent de remonter au plus tôt à la deuxième moitié du XIIe siècle, date de la composition de l'Historia Norwegia. Ce texte fait mention des Skralings, certainement des Inuit (McGovern 1985: 284; Faulkes \& Perkins 2001: 3) que les Scandinaves auraient rencontrés lors de leurs expéditions vers le nord. Supposer que le déplacement des Scandinaves vers le nord était motivé par la chasse est parfaitement légitime (Seaver 2010: 101).

Qu'en est-il du phoque? Il est chassé en Scandinavie. Parmi les produits que les Finnas paient aux Norvégiens en guise de tribut, Ohthere mentionne des cordages de bateaux faits avec des peaux de phoques: «seoles hyde» (Lebecq 2011b: 235). C'est une preuve que les Lapons le chassent au IXe siècle. De même, dans la Saga de saint Oláf, Snorri Sturluson évoque une station de pêche (útver) en Hålogaland, où l'on trouve des phoques (Boyer 2007: 267).

L'Islande est un autre site de chasse. Dans la Saga d'Egill, fils de Grimr le Chauve, Grímr s'installe dans les Mýrar et se nourrit en partie de phoques et de baleines échouées (Boyer 1987b: 54). D'après le sagnamaðr [singulier de sagnamenn, i.e. l'auteur de la saga], le pinnipède est présent sur l'île et susceptible d'y être tué. En tant que textes littéraires, les sagas doivent être appréhendées de manière critique; toutefois la chasse au phoque sur l'île boréale est bien attestée par l'archéologie, par exemple à Reykjavík (Perdikaris et al. 2002: 6, 7).

Enfin, le Groenland constitue une véritable réserve de phoques (Jónsson 1997: 69). L'archéozoologie nous est ici d'une grande utilité. Des os de phoque ont été découverts dans un grand nombre de fermes des Établissements (Perdikaris \& McGovern 2008: 197-198); en tant que déchets, ils témoignent de l'exploitation qu'en ont fait les colons et donc de leur chasse.

\section{TECHNIQUES ET DIFFICUlTÉS DE LA CHASSE}

Si la question de la localisation des lieux de chasse a été éclaircie, celle des techniques est plus complexe. Dans l'Historia de gentibus Septentrionalibus, Olaus Magnus livre une description fictive de la chasse au morse. Inspirée du De animalibus d'Albert le Grand (Moulinier 1992), la scène est presque épique: le morse escalade les falaises (Fig. 2) et, après s'être rassasié d'herbe, il tombe de fatigue et s'endort sur les rochers les bordant. À ce moment, les hommes détachent un bout de peau près de la queue de l'animal et le fixent fermement aux arbres et rochers environnants. Ils lancent des pierres sur la tête de l'animal pour le réveiller. Lorsque c'est chose faite, le monstre cherche à fuir, s'agite et se dépèce lui-même (Olaus Magnus 1998:, xxi, 28).

À la lecture de cette scène, l'exagération de la profondeur du sommeil de l'animal peut retenir notre attention. Le morse partage cette spécificité avec le phoque; un passage de l'Histoire naturelle de Pline l'Ancien décrit en effet ce dernier comme l'animal ayant le sommeil le plus profond (Pline l'Ancien 1955: 15), caractéristique que reprend Olaus Magnus (Olaus Magnus 1998: xx, 5). Si l'origine de la croyance concernant le phoque est attestée par Pline l'Ancien, le cas du morse n'est pas aussi évident. Olaus Magnus a peut-être été influencé par la Konungs skuggsjá:

"Il reste une espèce que les Groenlandais comptent parmi les baleines, mais qui me paraît plutôt devoir être classée parmi les phoques; son nom est rostungr [morse]. L'aspect de ce poisson est tout à fait comme celui d'un phoque [...], mais [...] il a deux grandes dents en plus des petites dents [...] situées devant dans la mâchoire supérieure" (Jónsson 1997: 69).

D'après le récit norvégien du XIIIe siècle, le morse est comparable au phoque. En fin de compte, Olaus Magnus, humaniste du XVIe siècle, a pu compiler à la fois les données extraites de Pline l'Ancien et du texte anonyme scandinave (Delliaux 2015:36, 39, 40). Sous sa plume, le rosmarus/morsus devient un animal au sommeil profond, ce qui constitue ici un atout pour les chasseurs.

Le dépeçage de l'animal est au cœur de la technique de chasse au morse présentée par Olaus Magnus. Ce n'est $a$ priori pas un hasard, étant donné que la peau de morse est utilisée par les hommes. Néanmoins, il faut garder à l'esprit que l'un des objectifs de l'auteur suédois est de faire du Septentrion un lieu d'étrangetés et d'exotisme (Johannesson 1991: 175-177). Cela devait permettre d'assurer le succès de son œuvre, ce qui passe notamment par des scènes pittoresques et des dessins très caractéristiques de monstres marins (Balzamo 2009: 122; Ribémont 1997: 102-103).

Finalement, sur quels éléments pouvons-nous appuyer notre réflexion? Grâce à l'archéologie, une lame de chert (silice, quartz), qui pouvait constituer un fer de lance, a été découverte enfoncée dans une côte de morse dans le site de Tayara au Canada (Monchot et al. 2013: 22-23). Cette découverte sort du contexte géographique défini pour notre travail, de même qu'elle est extérieure à son contexte chronologique, puisqu'elle date de l'époque paléo-esquimaude (Monchot et al. 2013: 17). Néanmoins, elle peut servir de point de départ, compte tenu des silences relatifs aux sites archéologiques de l'aire géographique de la présente étude. Des pratiques cynégétiques similaires avaient-elles cours en Scandinavie, en Islande ou au Groenland? 
Les sagas islandaises attestent l'utilisation du harpon pour chasser les animaux marins et amphibies. Dans la Saga des frères jurés, les gens du Groenland se rendent en Islande pour assister au ping (assemblée) de Garðar. Ils viennent avec leur équipement de chasse et de pêche, notamment un harpon à phoque: «selskutil» (Boyer 1987e: 696). Le sagnamadr veut paraître authentique et sème des détails. Ils sont intéressants pour nous, puisqu'ils révèlent en partie les pratiques à un moment donné (l'Âge viking), mais avec un regard postérieur (XIII siècle) et donc partiellement déformé. Le harpon apparait par ailleurs dans un toponyme du Landnámabók (relatif à l'Islande) : Skutilsfförðr, c'est-à-dire "Fjord-du-harpon " (Boyer 2000: 124). Ce toponyme désigne-t-il une zone de chasse au harpon ou un lieu où a été découvert un harpon perdu ou abandonné? Nous ne trancherons pas. Cela dit, retenons que le harpon, au vu de ces éléments, pouvait être utilisé pour la chasse.

La valeur historique de ces récits a déjà été discutée. L'utilisation du harpon est toutefois confirmée par la loi du Gulaping: "Si un homme blesse un phoque, celui qui le trouve peut le garder, mais il doit rendre le harpon à son propriétaire" (Larson 2008: 102). En Norvège, lieu de promulgation de la loi du Gulaping, le harpon semble donc être une arme privilégiée pour chasser les animaux marins et amphibies, en tout cas le phoque. Mais ces animaux ont une très épaisse couche de graisse (Monchot et al. 2013: 22), destinée à les protéger du froid de l'eau, de la neige/glace et de l'air. Dans ces conditions, le harpon est efficace, car il permet de transpercer cette couche de graisse. Les crochets de sa pointe permettent ensuite de maintenir l'arme dans la chair de l'animal.

Malgré l'utilité du harpon, d'autres techniques de chasse existent. Compte tenu de l'épaisseur de sa peau, utiliser un gourdin pour tuer le phoque peut s'avérer un choix judicieux (Perdikaris \& McGovern 2008: 194-197) : «Le veau marin $[\ldots]$ possède un corps charnu et dur, c'est pourquoi il est difficile à tuer si on ne lui brise pas les tempes" (Olaus Magnus 1998: xx, 4). L'appellation veau marin (vitulus marinus) nous vient de Pline l'Ancien (Pline l'Ancien 1552: 49). L'auteur romain constate en effet une ressemblance entre le phoque et le veau en raison de leur mugissement (Pline l'Ancien 1555: 13). Dans l'Historia, Olaus Magnus reprend cette dénomination lorsqu'il évoque la chasse au gourdin.

Le gourdin est en effet une autre technique de chasse, particulièrement appréciée par les Scandinaves installés au Groenland (Perdikaris \& McGovern 2008: 197). Ils ont pour proies privilégiées les espèces de phoque migrant au printemps. D'un côté, les femelles de deux espèces de phocidés, le phoque gris (Halichoerus gryphus (Fabricius, 1791)) et le phoque commun (Phoca vitulina Linnaeus, 1758), viennent sur la terre ferme au printemps, au moment de la mise bas. De l'autre, à la même saison, le phoque du Groenland (Pagophilus groenlandicus (Erxleben, 1777)) et le phoque à capuchon (Cystophora cristata (Erxleben, 1777)) sont présents sur les côtes occidentales du Groenland après avoir dérivé sur des glaces flottantes en provenance du Labrador, où ils se reproduisent (Diamond 2006: 364, 365; Perdikaris \& McGovern 2008: 195). En revanche, il semble que les Scandinaves ont rencontré des difficultés pour chasser le phoque annelé (Pusa hispida (Schreber, 1775)), qui est pourtant une espèce sédentaire et la plus répandue dans les eaux groenlandaises, susceptible d'apporter des ressources alimentaires non négligeables durant l'hiver. Les colons ont essayé de chasser les spécimens de cette espèce au harpon, mais les résultats ont été décevants (Perdikaris \& $\mathrm{McGov}-$ ern 2008: 197). Le comportement de l'animal est en effet problématique pour la chasse. Il vit sous la glace, remontant rarement à la surface pour respirer au moyen de trous creusés dans la glace, dont la largeur ne lui permet que de sortir la tête. La technique pour le chasseur consiste donc patienter près d'un trou. Lorsqu'il aperçoit un phoque, il le harponne, bien souvent à l'aveugle, compte tenu de la couche de neige à la surface de l'eau (Diamond 2006: 419-420). Néanmoins, si les Inuit ont su adopter cette technique, les Scandinaves n'y sont jamais parvenus. Ils s'exposaient de ce fait au risque d'insuffisance alimentaire, ce qui peut expliquer - en partie au moins - l'effondrement des sociétés scandinaves du Groenland (Diamond 2006: 420).

La chasse est rendue difficile par les caractéristiques biologiques des phoques et des morses, à savoir leur épaisse couche de graisse, ainsi que par le comportement de certaines espèces. À cela viennent s'ajouter les difficultés socio-juridiques dont nous avons déjà parlé. Les droits des individus peuvent entrer en contradiction avec le rekatháttr d'un côté et le silence juridique concernant les almenningar de l'autre, ce qui peut conduire à des conflits. D'après la loi de la Jónsbók, il existe des zones de chasse libres de tout contrôle, situées útan netlóg, au-delà d'une limite à partir de laquelle des filets peuvent être posés. Les hommes sont autorisés à y chasser en toute impunité: "Allir menn eigu at veiða fyrir útan netlóg at osekiu» (Schulman 2010: 274, 275). Le fruit de la chasse revient de droit aux chasseurs. Notons que la Jónsbók renseigne sur une technique cynégétique particulière, faisant intervenir des filets (Perdikaris \& McGovern 2008: 194-197); il s'agit donc de capturer l'animal.

Ces difficultés biologiques et socio-juridiques ont été accentuées au moment du petit âge glaciaire (c. (1300-) 1400-1850) qui a mis fin à l'optimum climatique qui durait depuis le VIIIe siècle. La répartition des glaces estivales entre l'Islande et le Groenland a été modifiée (Perdikaris \& McGovern 2008: 189-190) et les zones de chasse sont devenues plus difficiles d'accès. Par exemple, le détroit de Davis était fréquemment impraticable en raison des glaces flottantes, rendant les Norðrsetr inaccessibles. À terme, le petit âge glaciaire aurait participé de l'effondrement des colonies scandinaves du Groenland (Keller 2010: 4-5). Les flux commerciaux en provenance de l'île boréale, en particulier l'ivoire de morse, se seraient effondrés en raison des difficultés croissantes pour chasser. Pourtant, la peau et l'ivoire de morse sont des produits exportés du Groenland et importants pour les populations installées sur l'île (Frei et al. 2015: 445-446). 


\section{APRÈS LA CHASSE: EXPLOITER ET COMMERCER}

\section{LES DIFFÉRENTES EXPLOITATIONS DU MORSE ET DU PHOQUE}

Les deux pinnipèdes sont chassés pour leur viande. Lorsque les Norvégiens s'installent en Islande dans les années 870 (Holman 2003: 146), les mammifères marins et amphibies sont pour eux un moyen de subsistance non négligeable, le territoire sur lequel ils débarquent étant quasi désert (Stefánsson 2003: 210). Rapidement, les colons ont développé l'agriculture. Les similitudes entre les terres norvégienne et insulaire ont été un avantage pour eux (Roesdahl 1998b: 262-263; Rafnsson 2001: 118; ). De même, les oiseaux sont une ressource saisonnière intéressante (Perdikaris \& McGovern 2008: 194-195).

$\mathrm{Au}$ Groenland, la viande de phoque revêt une importance considérable. Les circuits de redistribution des carcasses de phocidés des sites littoraux vers les fermes isolées de l'intérieur du territoire, en dépit de la topographie accidentée de l'île, témoignent de l'importance vitale de cette viande (Perdikaris \& McGovern 2008: 197-198). Ne tombons pas dans le déterminisme pour autant: les colons scandinaves peuvent également pêcher et chasser des rennes, des ours ou des oiseaux (Roesdahl 1998b: 272). L'essentiel du Groenland étant prisonnier de la neige et de la glace, les perspectives agricoles sont réduites (Roesdahl 1998b: 271). Le temps qu'ils développent l'activité pastorale sur l'île, les Scandinaves ont donc consommé de la viande de phoque. Alan Outram a étudié le matériel archéozoologique des sites de Sandnes et Niaquussat, dans les Établissements de l'Ouest. La proportion des os de phoque par rapport aux autres animaux (bovins, caprins, rennes) est plus importante. À Sandnes, les os de phoque représentent $31 \%$ du matériel découvert, contre $85 \%$ à Niaquussat. Sandnes a un statut supérieur à Niaquussat, ce qui expliquerait cet écart selon l'auteur. La viande de phoque serait donc consommée en priorité par la population du site moins prestigieux (Outram 1999: 106-109).

La question de la consommation de la viande de morse au Moyen Âge est l'objet de discussions. Dans la Grágás, il est spécifié que l'animal ne peut être mangé qu'en temps de charnage, période de l'année où la consommation de viande est autorisée. Sa chair est donc considérée comme carnée.

"Quand un homme jeûne la nuit, il est autorisé à manger des aliments secs comme des plantes, fruits et tout ce qui pousse sur terre. Il peut également manger [...] du poisson de toutes sortes et des baleines, d'autres espèces que le morse et le phoque, ces dernières ne pouvant être mangées que lorsque la consommation de viande est autorisée" (Dennis et al. 1980: $50)$.

Cette interdiction s'explique peut-être par une contamination de la proscription de la viande de cheval à son équivalent marin, la baleine-cheval ou le morse (Dennis et al. 1980: 49; Larson 2008: 49). Peut-être aussi que le morse est proscrit car perçu comme ad malam partem (Lecouteux 1993: 56; Dierkens \& Gautier 2014: 13). Dans ce cas, cette proscription pourrait s'expliquer par la confusion qu'engendre la multi- tude de dénominations de l'animal, comme nous l'avons vu précédemment.

Mais ces animaux sont aussi exploités à d'autres fins. D'abord, leur peau peut être utilisée pour faire des cordages, ce qui a déjà été évoqué. Mais elle protège aussi du froid. Dans les sagas, on rencontre parfois des personnages avec des vêtements en peau de phoque à l'image de Loðinn avec sa cape et ses braies : "Loðinn var iselskinnstakki og selskinnsbrókum" (Boyer 1987e: 694). Ensuite, avec leur graisse ou leur huile, les hommes calfatent leurs embarcations. Dans la Saga d'Eirikr le Rouge, une barque goudronnée de graisse de phoque est mentionnée et, d'après le sagnamaðr, elle résiste mieux aux tarets, petits mollusques vermiformes s'attaquant au bois immergé (Boyer 1987c: 354). L'huile de mammifères marins et amphibies sert pour l'éclairage et a une valeur symbolique. Durant les nuits polaires, les jarls se doivent en effet de maintenir leur statut social, ce qui passe notamment par l'assurance d'un éclairage constant (Storli 2007: 93). Enfin, leur exploitation peut répondre à des besoins médicaux. D'après Olaus Magnus, le sang de phoque, mélangé à du lait de jument, était consommé pour soigner l'insomnie (Olaus Magnus 1998: xx, 5). Il s'agit d'une caractéristique relevant de ce que James G. Frazer (1984) a nommé la magie sympathique, c'est-à-dire fondée sur l'idée que le semblable appelle le semblable: au Ier siècle, Pline l'Ancien a mis en avant le sommeil profond du phoque, spécificité que reprend l'humaniste suédois au XVIe siècle, faisant du sang de phoque un remède contre l'insomnie (Frazer 1984).

\section{LE COMMERCE DE L'IVOIRE DE MORSE}

L'ivoire est «une variété de tissu osseux contenant du phosphate de calcium, du phosphate de magnésium et du carbonate de calcium, qui constituent la dentine» (Fuga 2005: 150). Au Moyen Âge, l'ivoire de morse est apprécié pour sa robustesse, ses vertus médicinales ou talismaniques, sa blancheur et sa pureté (Pastoureau 2004: 311). Les canines de l'animal sont extraites de sa mâchoire après avoir laissé la carcasse se décomposer quelques semaines, ce qui facilite le travail (McGovern 1990). Bien que facilitée par l'état avancé de décomposition, l'extraction des défenses peut toutefois endommager le matériau. En Islande, sur le site d'Aðalstræti, des traces d'outils ont en effet été remarquées sur des canines de morse (Pierce 2009: 57-58). Un instrument comparable à une pince a vraisemblablement été employé pour séparer la dent du reste de la mâchoire, marquant durablement l'ivoire. Le fait que cette défense ait été découverte en Islande, sur le lieu potentiel de chasse de l'animal, traduit peut-être son impossible commercialisation en raison de sa dégradation.

La qualité de l'ivoire de morse est inférieure à celle d'éléphant, les canines de morses étant plus petites et de section plus ovale que les défenses du pachyderme (Gaborit-Chopin 1992: 204), ce qui peut compliquer le travail des artisans. De même, il est moins blanc, d'une "blancheur jaunâtre" (Roesdahl 1998a: 12) et de moins bonne qualité. En raison de la faible épaisseur de la défense de morse, l'ostéo-dentine apparaît souvent sur l'objet travaillé, soit sur la tranche, soit sur le revers. Il s'agit de la substance ambrée et translucide qui compose la 
chambre pulpaire. Elle est agglomérée en grains irréguliers, les perles. Au-dessus de cette couche se trouve l'ivoire proprement dit. Il est recouvert par une troisième couche, le cément, un revêtement de nature osseuse (Gaborit-Chopin 1978: 12).

On peut observer ce genre de défaut sur une pièce des Lewis Chessmen (Stratford 2001: 26; Bardiès-Fronty et al. 2012: 80). Les perles de l'ostéo-dentine de la canine de morse apparaissent clairement sur le profil gauche d'une tour, le long du casque du personnage - un guerrier - symbolisant la pièce de jeu (Stratford 2001: 23). Le sculpteur, qui a vraisemblablement travaillé la pièce dans un atelier de Niðarós (Trondheim) en Norvège (Seaver 2010: 104; Bardiès-Fronty et al. 2012: 80), a toutefois su mettre à profit la faiblesse du matériau pour en faire un élément de décor du casque.

Toutes les pièces ne présentent évidemment pas cette caractéristique, à l'image de la pièce d'échecs du milieu du XIII siècle (Fig. 3) conservée au Musée d'art et d'archéologie Antoine Vivenel de Compiègne (département de l'Oise, région Hauts-de-France). L'objet $(6,8 \times 6,7 \times 3,8 \mathrm{~cm})$ a été sculpté en Angleterre, en France ou en Scandinavie. Il s'agit d'un tour mettant en scène un chevalier - peut-être saint Michel - combattant un dragon.

À côté de ces pièces de jeu, les textes mentionnent quelques productions en ivoire de morse. Dans la Saga d'Eirikr le Rouge, on trouve par exemple un couteau à manche en dent de morse: "hnif tannskestan". Le sagnamadr évoque également une ceinture faite de dents de morse: "tannbelti» (Boyer 1987c: 337, 340). Dans ces deux cas, il s'agit de surinterprétations du traducteur: la source ne permet pas de dire s'il s'agit en effet d'ivoire de morse. Il peut s'agir d'une volonté de l'auteur de la saga de mettre en avant des éléments exotiques pour reconstituer l'univers supposé des Scandinaves de l'Âge viking dans un souci de réalisme (Boyer 2012: 106, 107, 111). Néanmoins, l'archéologie a mis au jour plusieurs objets de la vie quotidienne en ivoire de morse, notamment des pièces de jeu, des plaques-boucles, des peignes et boutons, en Suède et au Groenland (Roesdahl 1998a: 22; Boyer 2001a: 87). Les objets cités dans la saga ont donc tout à fait pu exister.

Les Scandinaves utilisent donc l'ivoire, mais ils en font également le commerce. L'ivoire d'éléphant devenant rare dans la seconde moitié du IXe siècle (Gaborit-Chopin 1992: 204; Roesdahl 1998a: 18), celui de morse s'impose progressivement. La ville de Cologne a été particulièrement dynamique pour l'artisanat ivoirier (Bardiès-Fronty et al. 2012: 71, 81). Plusieurs pièces décrites comme ayant été travaillées dans cette ville ont été exposées au Musée de Cluny en 2012-2013 à l'occasion de l'exposition Art du jeu, jeu dans l'art. De la même manière, à partir de la fin du IXe siècle - date de la visite d'Ohthere à la cour du roi Alfred - se développe en Angleterre un style particulier de l'artisanat ivoirier: le Winchester style (Lebecq 2011c: 242-243). L'ivoire arrive donc brut et il est travaillé par les artisans, qui développent un courant artistique spécifique. Des échanges existent donc bien.

Toutefois, le commerce de l'ivoire de morse est antérieur à cette date. Le coffret de Gandersheim, ainsi nommé car découvert à Gandersheim (Basse-Saxe, Allemagne), est daté de

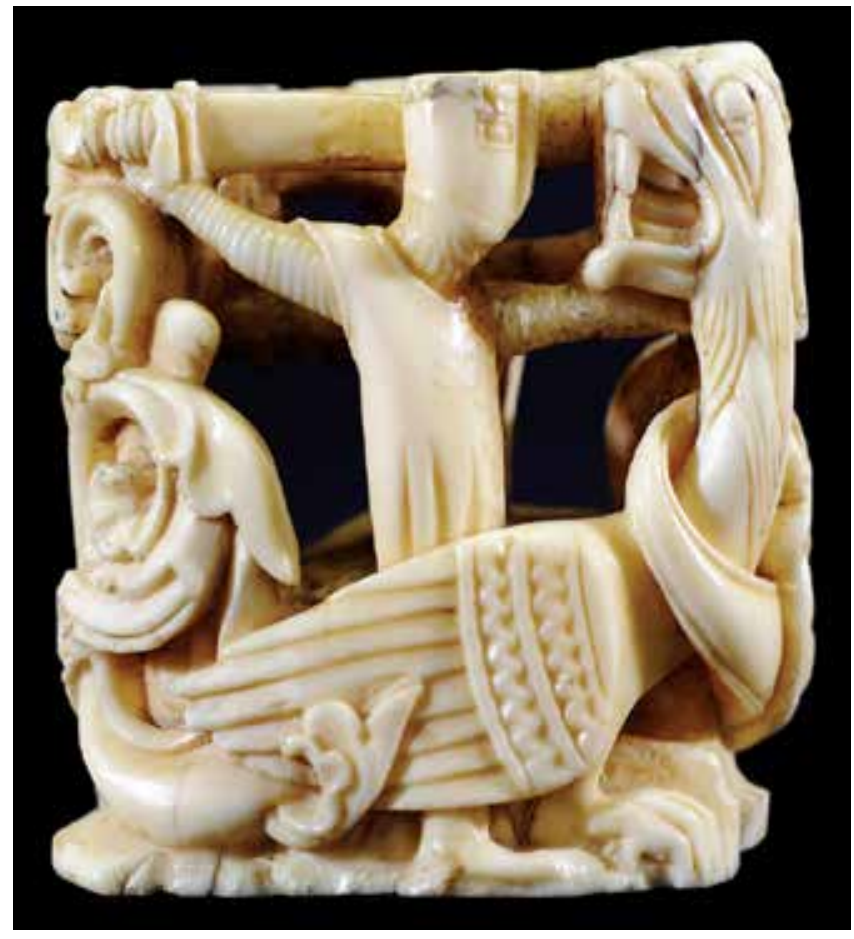

FIG. 3. - Pièce d'échecs (tour) en ivoire de morse (Angleterre, France ou Scandinavie, milieu XIIle siècle). Photo Benoît Roland, Musée Antoine Vivenel, Compiègne.

la fin du VIIIe siècle. L'objet est constitué de montures et d'un verrou de bronze, ainsi que de panneaux d'ivoire (Beckwith 1972: 118). En raison de l'inscription mentionnant la Vierge qui l'orne (du fait que le coffret abritait supposément une relique de Marie) et des similitudes entre les motifs dudit coffret et ceux de manuscrits anglais de la même époque (Beckwith 1972: 18), il est possible que l'objet ait été confectionné en Angleterre, la Scandinavie de la fin du VIIIe siècle étant qui plus est encore païenne (Holman 2003: 65).

Dans ce cas, l'ivoire du coffret pourrait provenir de morses chassés en Scandinavie. Il aurait alors été exporté sous sa forme brute jusqu'en Angleterre, où des artisans l'auraient travaillé. Un réseau d'échanges entre l'Europe et la Scandinavie, initié par les Européens, existe déjà à l'époque (Lebecq 2011a: 138). En retour, les Scandinaves, appâtés par les richesses du Continent, ont emprunté les routes de navigation en sens inverse. Il n'est pas impossible qu'ils aient emmené avec eux des défenses de morse, en guise de monnaie d'échange.

Le Diplomatarium Norvegicum, recueil des relations diplomatiques entre Britanniques et Scandinaves (Lenègre 2012: 207), fournit d'ailleurs un exemple précis de défenses arrivées brutes en Angleterre, pour être travaillées par les artisans locaux. Dans une lettre de 1224-1225 émanant du roi Håkon de Norvège (au nominatif dans le texte: Haquinus rex Norvegiae) et adressée au roi Henri III d'Angleterre (au datif dans le texte: Henrico regi Anglie), il est fait mention de dents de baleine: "dentes cetinas» (Diplomatarium Norvegicum Vol. 19, diplôme no 167). Pour Else Roesdahl, il peut tout à fait s'agir de défenses de morse (Roesdahl 1998a: 40). 


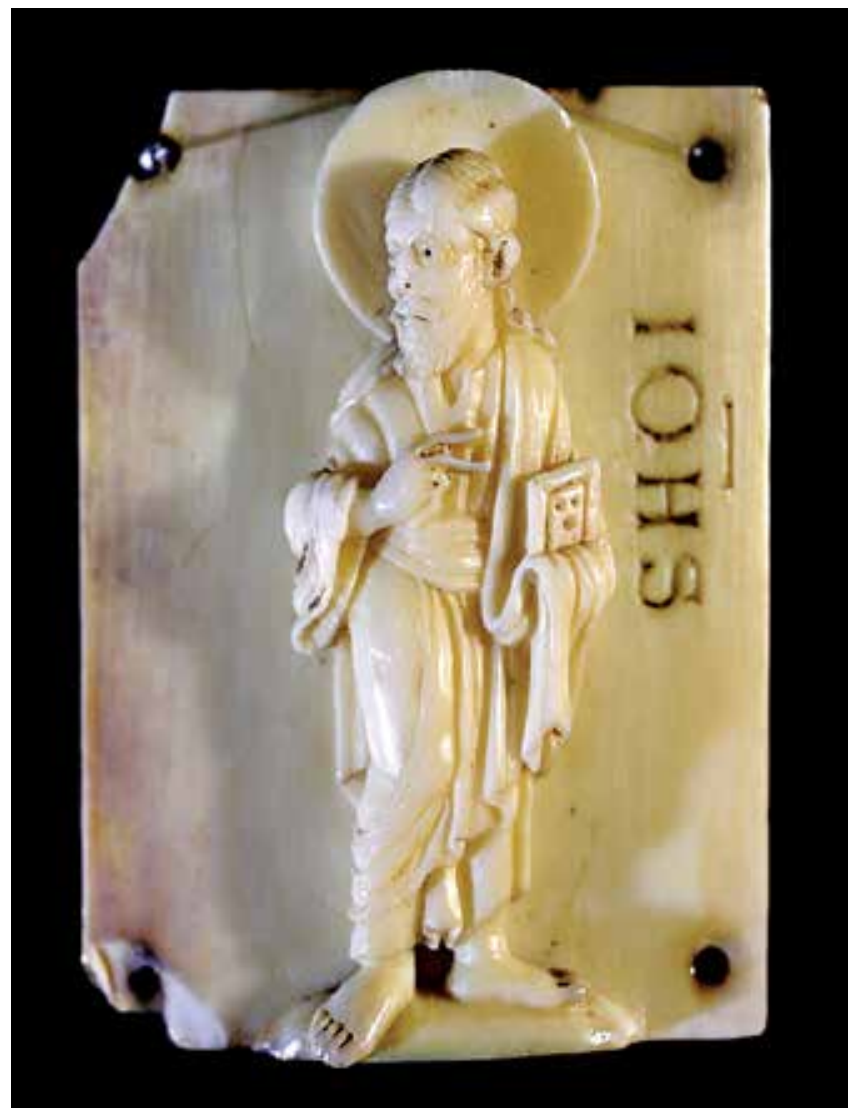

FIG. 4. - Autel portatif en ivoire de morse (Angleterre ou France septentrionale XIle siècle). Photo Benoît Roland, Musée Antoine Vivenel, Compiègne.

À partir de la seconde moitié du IXe siècle, les produits en ivoire de morse sont de plus en plus nombreux. Sur l'île de Groix, en Bretagne, des dés et pions ont été découverts en 1906 dans une sépulture viking datant du Xe siècle (Renaud 2000: 64-65). De plus, le Musée Antoine Vivenel possède dans sa collection médiévale un autel portatif $(4,5 \times 3,5 \mathrm{~cm})$ du XIIe siècle, sculpté en Angleterre ou en France septentrionale et représentant saint Jean, comme l'indique l'inscription IOHS, i. e. Iohannus (Fig. 4).

La valeur de l'ivoire ne faiblit pas durant les siècles suivant. En 1327, une embarcation avec une cargaison d'environ 520 défenses de morse, correspondant à six années de dîme du Groenland, débarque au port de Bergen en Norvège (Roesdahl 1998a: 44 ; Keller 2010: 5). Les marchands s'intéressent aux canines, qui sont vendues "douze livres, quatorze sous» et "six sous turon argenti" [sous tournois d'argent] (Roesdahl 1998a: 44). Ces deux sommes sont importantes et montrent que l'ivoire fait l'objet d'un commerce lucratif.

Mais la circulation de l'ivoire de morse ne s'explique pas uniquement par le commerce, puisque le matériau est aussi un précieux argument diplomatique. Donner ou recevoir un présent est un élément important dans les négociations (Bauduin 2009: 100). Par exemple, dans le Dit des Groenlandais, Sokki envoie son fils Einarr en Norvège dans le but d'instituer au Groenland un siège épiscopal. Einarr emporte avec lui des dents de morse, ainsi que des cordes en peau de morse, afin d'offrir des présents aux chefs qu'il sera amené à rencontrer (Boyer 1987a: 376).
Enfin, les crânes de morse sont appréciés au Moyen Âge en raison de leur exotisme. L'archéologie en a découvert à Dublin (Irlande), à Sigtuna (Suède) et à Schleswig (Allemagne). Le morse ne vit pas dans ces régions. Ainsi, un tel crâne y est un objet singulier et atypique, d'autant plus s'il est décoré, comme ceux découverts à Bergen (Norvège). Ils peuvent alors devenir des trophées exposés. À Oslo par exemple, a été découvert un crâne avec deux trous, permettant peut-être de suspendre l'objet à un mur (Roesdahl \& Stoklund 2006).

\section{CONCLUSION}

Au Moyen Âge, le phoque et le morse ont été des proies dans les mers du Nord. La chasse prend des formes diverses. Elle a lieu tantôt sur la terre ferme, tantôt en mer. De même, elle mobilise souvent un harpon, dont les textes se font l'écho, mais l'utilisation du gourdin ou du filet ne sont pas à négliger. Toutefois, les difficultés rencontrées par les chasseurs sont nombreuses, tenant en premier lieu aux caractéristiques biologiques de l'animal. À ces contraintes viennent s'ajouter des facteurs juridiques et climatiques, tout aussi problématiques.

La relation homme-animal reste ambiguë, caractérisée d'un côté par une nécessité - alimentaire par exemple - et de l'autre par une méfiance. L'exemple du hrosshvalr est significatif, au point qu'il aurait donné lieu à une interdiction alimentaire. L'animal est enfin une source potentielle d'enrichissement, en particulier le morse avec son précieux ivoire. L'inaccessibilité progressive des Norðrsetr et l'effondrement des flux commerciaux de ce matériau, véritable poumon économique pour les Scandinaves du Groenland, serait l'une des explications de la disparition de ces derniers de l'île.

L'ambition de cet article a été d'apporter quelques éléments de réponse sur la chasse, l'exploitation et le commerce du morse et du phoque dans les mers septentrionales au Moyen Âge. Le voile a en partie été levé grâce à l'étude de sources textuelles variées, d'une historicité inégale et apportant des regards différents. En les croisant, des particularités surgissent et permettent de mener une réflexion historique. Mais, comme cela a été annoncé dès l'introduction, les données archéologiques n'ont été utilisées que de manière secondaire et mériteraient d'être davantage mises en valeur.

\section{Remerciements}

Cet article reprend de nombreuses thématiques que j'ai eu l'occasion d'explorer durant mon Master Recherche (années universitaires 2013-2014 et 2014-2015) à l'Université du Littoral-Côte d'Opale (U.L.C.O.) de Boulogne-sur-Mer. Je tiens donc à remercier le département Histoire de cette université, ainsi que le Centre de Recherche en Histoire Atlantique Et Littorale (C.R.H.A.E.L.), pour m'avoir permis de mener à bien ce projet. J'adresse également mes plus chaleureux remerciements à mon directeur de recherches, Alban Gautier, maître de conférences en histoire médiévale et membre junior de l'Institut Universitaire de France. 
Merci également à Jean-Luc Bourgain, directeur du service dédié aux mammiferes marins du centre Nausicaà de Boulognesur-Mer. Il a témoigné un vif intérêt à l'égard cette étude et s'est appliqué à répondre à certaines de mes interrogations.

De plus, ma gratitude s'adresse à la Bibliothèque de l'Université du Littoral-Côte d'Opale (B.U.L.C.O.), à la Bibliothèque Nordique ainsi qu'à la Bibliothèque d'Anatomie Comparée (Museum national d'Histoire naturelle), qui m'ont accueilli et m'ont offert le loisir de consulter des ouvrages indispensables à ma réflexion.

Enfin, merci aux relecteurs anonymes d'Anthropozoologica pour leurs commentaires et suggestions, ainsi qu'à tous ceux qui, pour des raisons pratiques, ne peuvent pas être mentionnés ici mais qui n’ont eu de cesse de me soutenir.

\section{RÉFÉRENCES}

\section{SOURCES}

BOYER R. 1987a. - Dit des Groenlandais, in Boyer R. (trad.), Sagas islandaises. Gallimard, Paris: 376-388.

BOYER R. 1987b. - Saga d'Egill, fils de Grímr le Chauve, in Boyer R. (trad.), Sagas islandaises. Gallimard, Paris: 3-203.

Boyer R. 1987c. - Saga d'Eiríkr le Rouge, in Boyer R. (trad.), Sagas islandaises. Gallimard, Paris: 331-355.

Boyer R. 1987d. - Saga de Snorri le Godi, in Boyer R. (trad.), Sagas islandaises. Gallimard, Paris: 205-330.

BOYER R. 1987e. - Saga des frères jurés, in Boyer R.. (trad.), Sagas islandaises. Gallimard, Paris: 637-718.

BOYER R. (trad.), 2000. - Le livre de la colonisation de l'Islande. Brepols, Turnhout, $384 \mathrm{p}$.

Boyer R. (trad.)2007. - La Saga de saint Oláf de Snorri Sturluson. Payot \& Rivages, Paris, 496 p.

Dennis A., FoOTE P. \& PERKIns R. M. (trad.), 1980. — Laws of Early Iceland: Grágás I. Winnipeg, University of Manitoba Press, $288 \mathrm{p}$.

DIPLOMATARIUM NORVEGICUM, XIX. http://www.dokpro.uio.no/dipl_ norv/diplom_field_eng.html dernière consultation : 11/08/2016.

JónSSON E. M. (trad.) 1997. - Introduction, in Le Miroir royal. Paris, Esprit Ouvert:13-23.

LARSON L. M. (trad.), 2008. - The Earliest Norwegian Laws. Being the Gulathing and the Frastathing Law. Clark, The Lawbook Exchange, ix $+451 \mathrm{p}$.

MAILlefer J.-M. 2004. - Introduction, in Olaus Magnus, Histoire et description des peuples du Nord. Les Belles Lettres, Paris: 9-16.

Olaus Magnus 1998. - Historia de gentibus Septentrionalibus Vol. III, Livres XVI-XXII. Fisher P. \& HigGens H. (trads). The Hakluyt Society, Londres, v + 771-1247.

PLINE L'ANCIEN 1952. - Histoire naturelle, Livre VIII (des animaux terrestres). ERnOUT A. (trad.). Paris, Les Belles Lettres, 267 p.

PLINE L'ANCIEN 1955. - Histoire naturelle, Livre IX (des animaux marins). SAINT-DENIS E. DE (trad.). Paris, Les Belles Lettres, 224 p.

SCHULMAN J. K. (trad.) 2010. - Jónsbók, the Laws of Later Iceland. The Icelandic Text according to MS AM 351 fol. Skálholtsbók eldri. AQ-Verlag, Saarbrücken, xxxii +485 p.

ARTICLES ET OUVRAGES

BALZAMO E. 2009. - Olaus Magnus savait-il dessiner? Quelques réflexions et hypothèses au sujet des vignettes de l'Historia de gentibus Septentrionalibus. Proxima Thule 6: 121-141.

BARBARINI F. 1990. — Recherche et emploi des richesses parmi les Scandinaves à l'âge des Vikings. Cahier des Annales de Normandie 23 (1): 71-87.

BardiÈs-Fronty I., Boehm B., DunN-VATURi A.-E., Finkel I., Goret J.-F., GRANDET M., Lepape S., LeRAT M., SCHÄDler U. \& TARROUX S. 2012. - Art du jeu, jeu dans l'art: de Babylone à l'Occident médiéval. Réunion des musées nationaux; Grand Palais, Paris, 159 p.
BAUduIn P. 2009. - Le monde franc et les Vikings. Albin Michel, Paris, 455 p.

BECKWITH J. 1972. - Ivory Carvings in Early Medieval England. Harvey Miller, Londres, 168 p.

BOYER R. 2001a. - L'art viking. La Renaissance du livre, Tournai, 205 p. BOYER R. 2001b. - L'Islande médiévale. Les Belles Lettres, Paris, $272 \mathrm{p}$.

BOYER R. 2012. - Snorri Sturluson, le plus grand écrivain islandais du Moyen Âge. OREP, Bayeux, 160 p.

BYOCK J. 2007. - L'Islande des Vikings. Aubier, Paris, 492 p.

CATTANÉO G. 2013a - Codes juridiques. http://www.menestrel.fr/spip. php? rubrique $1868 \&$ lang=fr dernière consultation 12/08/2016.

CATTANÉO G. 2013b - Historiographie. http://www.menestrel.fr/spip. php?rubrique1869\&lang=fr dernière consultation 12/08/2016.

CHARPENTIER LJUNGQVIST F. 2005. - The significance of remote resource regions for Norse Greenland. Scripta Islandica 56: 13-54.

COllignon B. 1996. - Les Inuit. Ce qu'ils savent du territoire. L'Harmattan, Paris, $254 \mathrm{p}$.

Corbin A. 2004. - Introduction, in CORBIN A. \& Richard H. (dir.), La mer, terreur et fascination. BnF; Seuil, Paris, p. 13-13.

DelliauX M. 2015. - La transmission du savoir sur les monstres marins: l'exemple d'Olaus Magnus au XVIe siècle, in CLAUZEL I. (dir.), Langage et communication dans le Nord de la France à travers le temps. Cercle d'Études en Pays Boulonnais, Boulogne-sur-Mer: 31-47.

DeLORT R. 1984. - Les animaux ont une histoire. Seuil, Paris, 397 p.

Delort R. 1999. - Animaux, in Le Goff J. \& SCHMitT J.-C. (dir.), Dictionnaire raisonné de l'Occident médiéval. Fayard, Paris: 55-66.

DiAMOND J. 2006. - Effondrement: comment les sociétés décident de leur disparition ou de leur survie. Gallimard, Paris, 648 p.

DieRKENS A. \& GAUTIER A. 2014. - Inmundum atque exsecrabile: retour sur la question de l'hippophagie dans l'Europe du Nord et du Nord-Ouest au haut Moyen Age. Colloque La viande: fabrique et représentations, Tours, 29 novembre-12 décembre 2012.

DiLLMANN F.-X. 2006. - Les magiciens dans l'Islande ancienne. Études sur la représentation de la magie islandaise et de ses agents dans les sources littéraires norroises. Kungl. Gustav Adolfs Akademien för svensk folkkultur, Uppsala, xxi $+779 \mathrm{p}$.

Duchet Suchaux G. \& Pastoureau M. 2002. - Le bestiaire médiéval: dictionnaire historique et bibliographique. Le Léopard d'or, Paris, 163 p.

FAulKes A. \& PERKINS R. (eds.) 2001. - A history of Norway and the passion and miracles of the blessed Óláfr. Viking Society for Northern Research Text Series 13. http://www.vsnrweb-publications. org.uk/Text $\% 20$ Series/Historia $\% 26$ Passio.pdf dernière consultation: $12 / 08 / 2016$.

FlandRIn J.-L. \& MONTANARI M. 1996. — Histoire de l'alimentation. Fayard, Paris, 926 p.

FrAZER J. G. 1984. - Le rameau d'or. BELMONT N. \& IZARD M. (prefs). Robert Laffont, Paris, 4 vol.

Frei K. M., Coutu A. N., Smiarowski K., Harrison R., Madsen C. K., ARneborG J., Frei R., GUĐMUNDSSON G., SindBÆK S. M., WoOllett J., Hartman S., Hicks M. \& McGovern T. H. 2015. - Was it for walrus? Viking Age settlement and medieval walrus ivory trace in Iceland and Greenland. World Archaeology 47 (3): 439-466. http://dx.doi.org/10.1080/00438243.2015.1025912

Fuga A. 2005. - Techniques et matériaux des arts. Hazan, Paris, 384 p.

Gaborit-Chopin D. 1978. - Ivoires du Moyen Âge. Office du livre, Fribourg, 232 p.

GABORIT-CHOPIN D. 1992. - Le travail de l'ivoire de morse pendant la période romane, in RoesDahl E., MOHEN J.-P. \& DillmanN F.-X. (dir.), Les Vikings. . Les Scandinaves et l'Europe 800-1200: 22 exposition d'art du Conseil de l'Europe, Grand Palais, Paris, 2 avril12 juillet 1992, Altes Museum, Berlin, 1er septembre-15 novembre 1992, Nationalmuseet, Copenhague, 26 décembre 1992-14 mars 1993. Association française d'action artistique, Paris: 204-205.

Holman K. 2003. - Historical dictionary of the Vikings. Historical Dictionaries of Ancient Civilizations and Historical Eras Book 11. The Scarecrow Press, Lanham, 404 p. 
Horrent J. 1946. - À propos de "Gallerous". Localisation de la "Folie Tristan" de Berne, Gallerous et Rohal. Le Moyen Âge 52 (1): 43-72.

I.N.P.N. (INVENTAire NATIONAL DU PatRIMOINE NaTUREL). https:// inpn.mnhn.fr/accueil/index dernière consultation 13/08/2016.

JOHANNESSON K. 1991. - The Renaissance of the Goths in the Sixteenth-Century Sweden: Johannes and Olaus Magnus as Politicians and Historians. University of California Press, Berkeley, Los Angeles, Oxford, $298 \mathrm{p}$

Keller C. 2010. - Furs, fish and ivory. Medieval Norsemen at the Arctic Fringe. Journal of the North Atlantic 3 (1): 1-23. http:// dx.doi.org/10.3721/037.003.0105

LEBECQ S. 1983. - Marchands et navigateurs frisons du haut Moyen Âge, Vol. I: Essai. Presses Universitaires de Lille, Villeneuve d'Ascq, $470 \mathrm{p}$.

LEBECQ S. 2011a. - Aux origines du phénomène viking: quelques réflexions sur la part de responsabilité des Occidentaux (VIIIedébut IXe siècle), in Hommes, mers et terres du Nord au début du Moyen Agre, Vol. I: Peuples, cultures, territoires. Presses Universitaires du Septentrion, Villeneuve d'Ascq: 131-140.

LEBECQ S. 2011b. - Ohthere et Wulfstan: deux marchandsnavigateurs dans le Nord-Est européen à la fin du IXe siècle, in Hommes, mers et terres du Nord au début du Moyen Âge, Vol. I: Peuples, cultures, territoires. Presses Universitaires du Septentrion, Villeneuve d'Ascq: 223-237.

LEBECQ S. 2011c. - Scènes de chasse aux mammiferes marins (mers du Nord, VIe-XIIe siècles), in Hommes, mers et terres du Nord au début du Moyen Age, Vol. I: Peuples, cultures, territoires. Presses Universitaires du Septentrion, Villeneuve d'Ascq: 239-252.

LeCouteuX C. 1993. - Les monstres dans la pensée médiévale européenne. Presses de l'université Paris-Sorbonne, Paris, 256 p.

LENĖGRE F. 2012. — Norvégiens et Britanniques: identités et altérités au sein d'une Méditerranée septentrionale (XIIe-XIVe siècle), in Gautier A. \& Rossignol S. (éds), De la mer du Nord à la mer Baltique: identités, contacts et communications au Moyen Âge. Institut de Recherches Historiques du Septentrion, Villeneuve d'Ascq: 205-220.

Malaurie J. 1989. - Les derniers rois de Thulé. Plon, Paris, 655 p. MAREZ A. 2007. - Anthologie runique. Les Belles Lettres, Paris, 544 p.

MCGOVERN T. H. 1985. - The Arctic Frontier of Norse Greenland, in Green S. W. \& Perlman S. M. (éd.), The Archaeology of Frontiers and Boundaries. Academic Press, New-York: 73-122.

MCGOVERN T. H. 1990. - Walrus tusks \& bones from Aðalstrati 14-18, Reykjavík Iceland. NORSEC Report 55, $11 \mathrm{p}$.

McGovern T. H., Amorosi T., Perdikaris S. \& Woollett J. 1996. - Vertebrate zooarchaeology of Sandnes V51 : economic change at a chieftain's farm in west Greenland. Arctic Anthropology 33 (2): 94-121.

Monchot H., Houmard C., Dionne M.-M., Desrosiers P. M. \& Gendron D. 2013. - The Modus Operandi of Walrus exploitation during the Palaeoeskimo period at the Tayara site, Arctic Canada. Anthropozoologica 48 (1): 15-36.

Moulinier L. 1992. — Les baleines d'Albert le Grand. Médiévales (22-23): 117-128.

Ogilvie A. E. J., Woollett J. M., SMiarowski K., Arneborg J., Troelstra S., Kuijpers A., PÁlsdóttir A. \& McGovern T. H. 2009. - Seals and Sea Ice in medieval Greenland. Journal of the North Atlantic (2): 60-80.

OLSEN B. 2003. - Belligerent chieftains and oppressed hunters? Changing conceptions of interethnic relationships in Northern Norway during the Iron Age and Early Medieval period, in Barrett J. H. (éd.), Contact, Continuity and Collapse. The Norse Colonization of the North Atlantic. Brepols, Turnhout: 9-31.
Outram A. K. 1999. - A comparison of Paleo-Eskimo and medieval Norse bone fat exploitation in western Greenland. Arctic Anthropology (36): 103-117 https://www.jstor.org/stable/40316508

Pastoureau M. 2004. - Une histoire symbolique du Moyen Âge occidental. Seuil, Paris, 240 p.

Pastoureau M. 2013. - Le cochon. Gallimard, Paris, 128 p.

Perdikaris S. \& McGovern T. H. 2008. - Codfish and kings, seals and subsistence. Norse marine resource use in the North Atlantic, in Rick T. C. \& ERLANDSON J. M. (éd.), Human Impacts on Marine Environments. University of California Los Angeles Press, New York: 193-216.

PerdikARIS S., AMUNDSEn C. \& MCGOVERn T. H. 2002. - Report of Animal Bones from Tjarnargata 3C, Reykjavik, Iceland. NORSEC Zooarchaeology Laboratory Reports (1), 64 p.

PIERCE E. 2009. - Walrus hanting and the ivory trade in early Iceland. Archaeologia Islandica (7): 55-63.

RAFNSSON S. 2001. - The Atlantic islands, in SAWYER P. (éd.), The Oxford Illustrated History of the Vikings. Oxford University Press, New York: 110-133.

ReIJNDERS P., BRASSEUR S., VAN DER TOORN J., VAN DER WOlf P., Boyd I., Harwood J., Lavigne D. \& Lowry L. 1993. - Seals, Fur Seals, Sea Lions, and Walrus: Status Survey and Conservation Action Plan. IUCN, Gland, vii +88 p.

Renaud J. 2000. - Les Vikings en France. Ouest-France, Rennes, $125 \mathrm{p}$.

RiBÉMONT B. 1997. - L'inconnu géographique des encyclopédies médiévales: fermeture et étrangeté. Cahiers de Recherches Médiévales (3): 101-111.

ROESDAHL E. 1998a. - L'ivoire de morse et les colonies norroises du Groenland. Proxima Thulé (3): 9-48.

Roesdahl E. 1998b. - The Vikings. Pinguin Books, Londres, 352 p.

ROESDAHL E. \& STOKLUND M. 2006. - Un crâne de morse décoré et gravé de runes: à propos d'une découverte récente dans un musée du Mans. Proxima Thulé (5): 9-38.

SEAVER K. A. 2010. - The Last Vikings: The Epic Story of the Great Norse Voyages. I. B. Tauris, Londres, 288 p.

SHIRIHAI H. \& JARRETT B. 2011. - Guide des mammiferes marins $d u$ monde. Delachaux et Niestlé, Paris, 384 p.

SirräInen A. 2003. - The Stone and Bronze Ages, in Helle K. (éd.), The Cambridge History of Scandinavia. Cambridge University Press, Cambridge: 43-59. https://doi.org/10.1017/ CHOL9780521472999

STEFÁNSSON M. 2003. - The Norse Island Communities of the Western Ocean, in Helle K. (éd.), The Cambridge History of Scandinavia. Cambridge University Press, Cambridge: 202-220 http://dx.doi.org/10.1017/CHOL9780521472999.013

STORLI I. 2007. - Ohthere and his world. A contemporary perspective, in BAtely J. \& Englert A. (éd.), Ohtherés Voyages. A

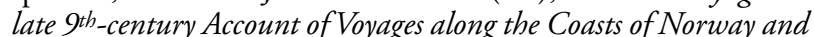
Denmark and its Cultural Context. The Viking Ship Museum, Roskilde: 76-99.

STRATFORD N. 2001. - The Lewis Chessmen and the Enigma of the Hoard. The British Museum, Londres, 64 p.

Thomasset C. 2002. - De la Bible à Albert le Grand, in JamesRaOul D. \& ThOmasset C., Dans l'eau, sous l'eau: le monde aquatique au Moyen Âge. Cultures et civilisations médiévales 25: 59-78.

Valtonen I. 2007. - Who were the Finnas? in BATEly J. \& ENGLERT A. (éd.), Ohthere's Voyages. A late 9th-century Account of Voyages along the Coasts of Norway and Denmark and its Cultural Context. The Viking Ship Museum, Roskilde: 106, 107.

VOISENET J. 2000. - Bêtes et Hommes dans le monde médiéval. Le bestiaire des clercs du Ve au XIIe siècle. Brepols, Turnhout, $535 \mathrm{p}$. 